\title{
Tribological tests of steel on polyamide 66 , polyamide 46 type surface contacts
}

\author{
Radu Papuc ${ }^{1}$, Cătălin Gavrilă ${ }^{2}$ Mihai - Tiberiu Lateș ${ }^{3}$ \\ ${ }^{1}$ Transilvania University of Brasov, radu.papuc@unitbv.ro, Romania \\ ${ }^{2}$ Transilvania University of Brasov, cgavrila@unitbv.ro, Romania \\ ${ }^{3}$ Transilvania University of Brasov, latesmt@unitbv.ro, Romania
}

\begin{abstract}
Improving the efficiency of mechanical transmissions is made, mostly, by finding new materials characterized through antifriction properties, specific reduced mass, wear resistance and by keeping the mechanical properties at high working temperatures (until $220{ }^{\circ} \mathrm{C}$ ). The paper presents the experimental tests for the surface type contacts between a steel made pin and different types of polyamides; finally there are presented the processing of the experimental data.
\end{abstract}

\section{Introduction}

The need of identifying the solutions for friction reduction between the components of the mechanical transmissions in relative motion, as well as the reduced wear, has led to researched by universities, research institutes and specialized companies to create new materials with antifriction properties; in this sense, the polyamides is a class of materials that answer to these demands - especially the groups: Polyamide 66(PA66) and Polyamide 46 (PA46), T. D. Mehnmet, D. Hayrettin, Y. Rifat [1]. Through research, in the first step there were developed the polyamides PA66 being characterized by high rigidity, low dimension variations with temperature variation, abrasion resistance, low dimension variations in high load case (because of high Young module values). These polyamides are used in the case of high loads (pressure) and temperatures up to 100 $\left({ }^{\circ} \mathrm{C}\right)$, M. W. Shin, S. S. Kim, H. Jang, [2]. Considering the exploitation conditions, it appeared the necessity to improve the antifriction properties, wear resistance and keeping the mechanical properties at temperatures up to $220\left({ }^{\circ} \mathrm{C}\right)$; in this manner, it was developed, by deep research, the PA46 polyamide class which keeps its characteristics up to these high temperatures and are characterized, at these temperatures, by friction coefficients of contact with steel components lower than the polyamides PA66, J. Van Ruiten, R. Proost, M. Meuwissen [3]. Lately, by recent research, there were brought notable improvements to polyamides by adding graphite to their composition and Teflon. By adding graphite in the internal composition of polyamides PA46 there will be obtained materials with specific reduced mass, high mechanical resistance, self lubricating properties (due to graphite), shock and vibration amortization properties, corrosion resistance, technological simplicity and reduced fabrication costs. Adding Teflon is the main concern of the polyamide producing companies with direct application in automobiles industry, with good antifriction properties and wear resistance at high temperatures (up to $220{ }^{\circ} \mathrm{C}$ ), specific low mass, and keeping the material properties at these temperatures, K. Randall, B. R. Antoni [4].

\section{Experimental determination of the friction coefficient}

For determining the friction coefficient between a steel pin and 3 types of polyamides (PA66, PA46, Teflon modified PA46) it will be used the UMT tribometer, presented in figure 1 which is equipped with the rotary module presented in figure 2 .

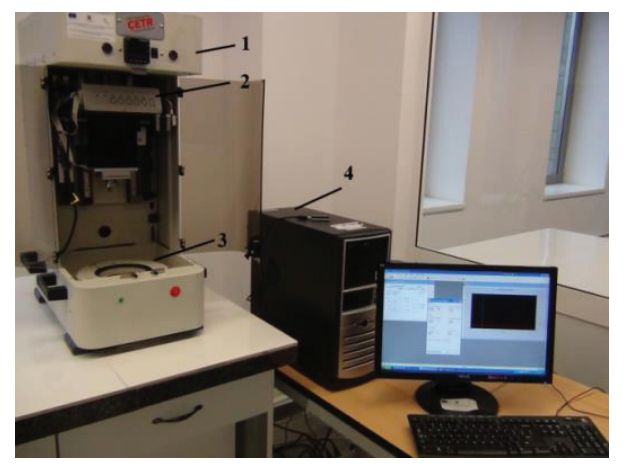

Fig.1. Tribometer UMT: 1 - command engine; 2 - positioning device; 3 - positioning and fixation rim; 4 - computer.

\footnotetext{
*Corresponding author: radu.papuc@unitbv.ro
} 
This tribometer has the following characteristics: it allows making the mechanical and tribological determinations for different types of materials; the measured physical quantities are processed by data processing software and then there are presented as diagrams.

In Fig. 1, are identified the principal components: the command motor and the positioning devices.

The UMT tribometer is connected to the computer through data cables.
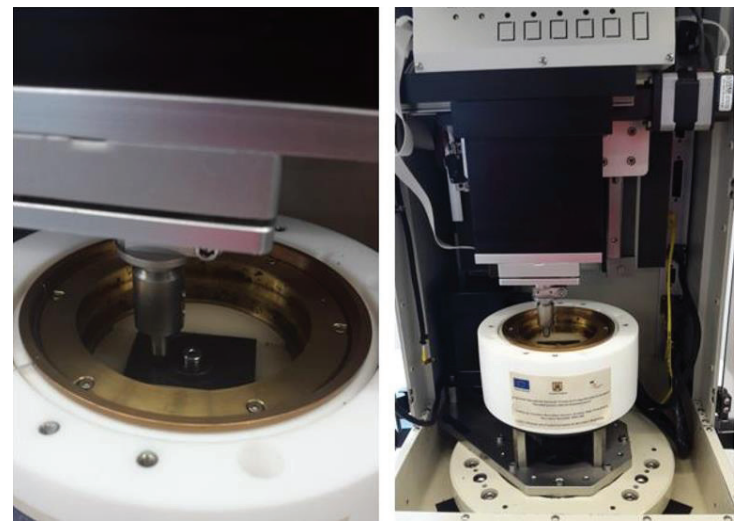

Fig. 2. The rotation module.

The PA66, PA46 and, respectively Teflon modified PA46 materials are used as plates, presented in figure 3 and mounted on a disk which is presented in figure 4.

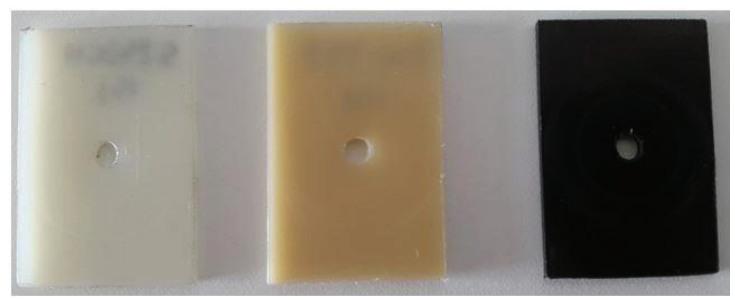

Fig. 3. The PA66, PA46 and, respectively Teflon modified PA46 plates.

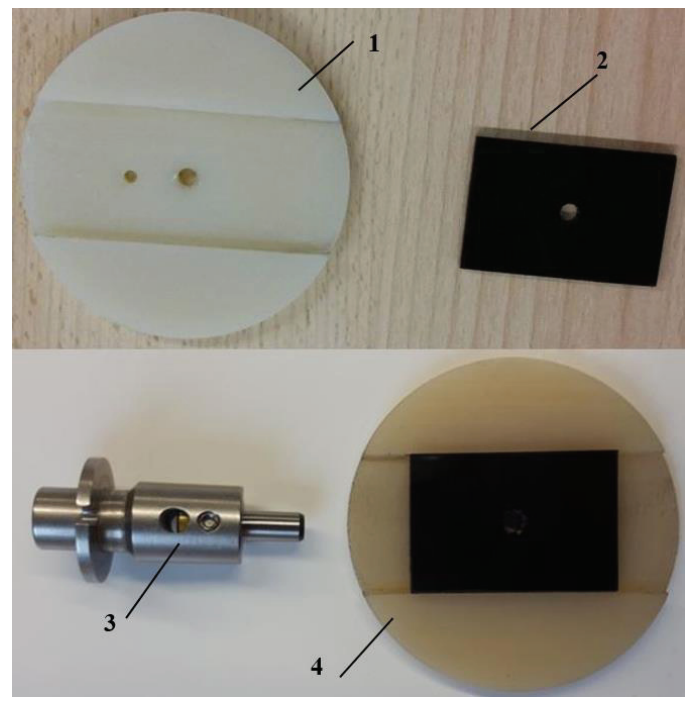

Fig. 4. The plates mounted on the disk, where: $1-$ the disk; 2 - the plates; 3 - the pin; 4 - the assembly.
The disk is mounted inside the tribometer rotation module and the contact is made between the normal pressing force pin and the PA66, PA46 and, respectively Teflon modified PA46 plates presented in the figure 4 .

In order to study in comparison the tribological properties of the three polyamides, in the following steps, there are performed tests in lubricated conditions, at a test environment temperature of $\mathrm{T}=120\left({ }^{\circ} \mathrm{C}\right)$, for the peripheral speeds $\mathrm{v}=1,6(\mathrm{~m} / \mathrm{s})$ and $3,2(\mathrm{~m} / \mathrm{s})$ in the conditions of a normal pressing force $F=7(\mathrm{~N})$. The friction coefficient values are presented in the figure 5 .

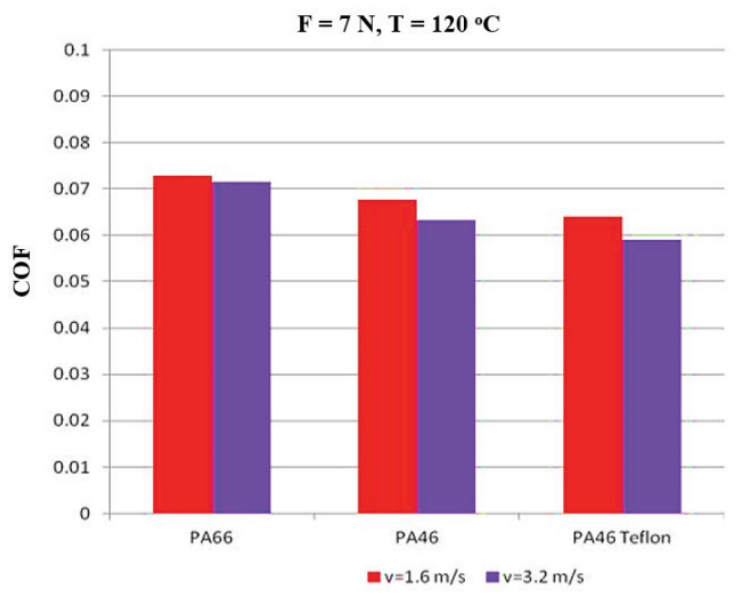

Fig. 5. The friction coefficient.

The friction coefficient value, for all polyamides, drops when the peripheral speed is increasing; the lowest values of the friction coefficient are in the case of Teflon modified PA46 polyamide.

Figure 6 presents, in percentages, the difference, by dropping down, of the friction coefficients for PA46 and Teflon modified PA46 polyamides relative to the PA66 polyamide friction coefficient, determined for the normal pressure force $\mathrm{F}=7(\mathrm{~N})$, peripheral speed $\mathbf{v}=3,2(\mathrm{~m} / \mathrm{s})$ and the temperature $\mathrm{T}=120\left({ }^{\circ} \mathrm{C}\right)$.

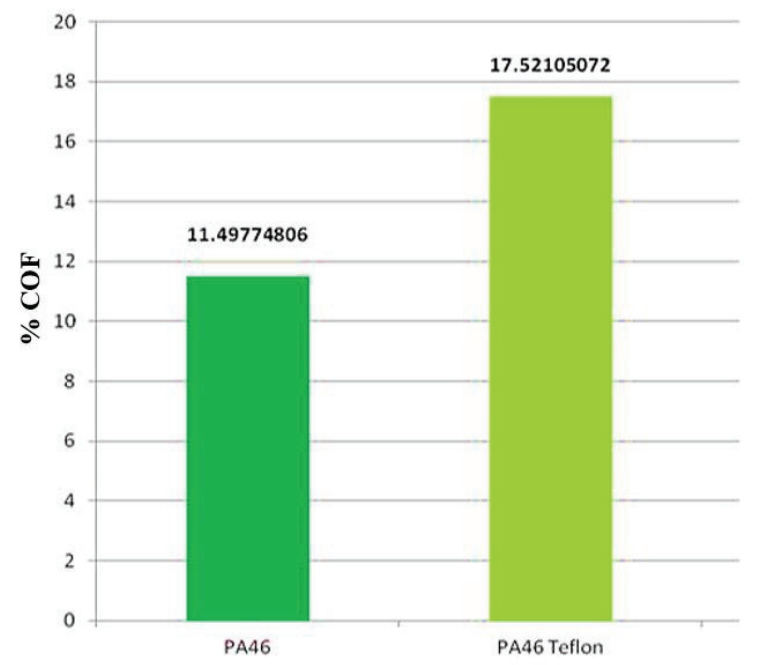

Fig. 6. The difference of friction coefficients to PA66; $F=7 \mathrm{~N}$, $\mathbf{v}=3,2 \mathrm{~m} / \mathrm{s}, \mathrm{T}=120^{\circ} \mathrm{C}$.

$\overline{{ }^{*} \text { Corresponding author: radu.papuc@unitbv.ro }}$ 
As it can be seen, the PA46 polyamide has the friction coefficient with 11,5 (\%) lower than the PA66 friction coefficient, and the Teflon modified PA46 polyamide has the friction coefficient with 17,5 (\%) lower than PA66; the percentage difference to the PA46 friction coefficient is $6.8(\%)$.

\section{Experimental processing data}

Measuring the physical quantities implies multiple measurements of the same quantity in the same testing conditions. The measurement process generates a random value set for the same physical quantities which can lead to wrong conclusions regarding the physical phenomena reproduced by testing.

The random value set resulted by measurement in the same conditions can have as fault the lack of control over some variables which affect the measurement process or a reduced precision for the measurement system.

The question is, for a value set obtained through $n$ measurements, which from these values can be kept as "good" values and which of these values must be eliminated as "bad". For example, it is considered as study the previously shown data, and as method used for data selection was chosen the modified Thompson $\tau$ technique from the document Measurement Uncertainty -American Society of Mechanical Engineers - ASME, 1998.

For the tests between the steel stud and the three polyamides (PA66, PA46 and Teflon modified PA46) there were made 5 measurements. By applying the modified Thompson $\tau$ method, the steps are presented as it follows.

For each of the 5 value sets there is calculated their average

$\overline{\mathbf{x}}=\frac{1}{\mathbf{n}} \sum_{\mathbf{i}=1}^{\mathbf{n}} \mathbf{x}_{\mathbf{i}}$

with $n=5$ representing the tests number performed on the same type of materials, in the same test conditions and $x_{\mathrm{i}}$ the measured value of the friction coefficient of test $i$ from the same set.

Next, for each of the $n=5$ value sets it is calculated their standard deviation with the relation.

$$
S=\sqrt{\frac{1}{n-1} \sum_{n-1}^{n}\left(x_{i}-\bar{x}\right)^{2}}
$$

and, further it is arranged inside each of the $n=5$ value sets, the measured values in increasing order; the minimum and maximum values are likely to be eliminated from the set.

For the minimum and maximum values measured in every set, the value deviation is calculated with the relation. $\delta_{\mathbf{m}}=\left|\mathbf{x}_{\min }-\overline{\mathbf{x}}\right|$

for the minimum value deviation and

$$
\delta_{\mathbf{M}}=\left|\mathbf{x}_{\min }-\overline{\mathbf{x}}\right|
$$

for the maximum value deviation; it is chosen

$$
\delta_{\text {Max }}=\max \left(\delta_{m}, \delta_{M}\right)
$$

Next, according to the numbers of the measurements on the same material, in the same conditions (in this case $\mathrm{n}=5$ ) from the document Measurement Uncertainty it is chosen the value for $\tau$ parameter - for $n=5, \tau=1,572$, R. Velicu, M. T. Lateș, G. Moldovean [5], for $n=4$, $\tau=1,393$ and for $n=3, \tau=1,150$, A. J. Wheeler, A. R. Ganji, [6].

If the condition

$$
\delta_{\text {Max }}>\tau S
$$

is accomplished, then the measured value corresponding to the maximum deviation is eliminated. The steps $1 \ldots 6$ are retaken until the condition 6 is no longer accomplished and, in this case, the accepted measured value is considered to be equal with the mean calculated with the value 1 .

After applying this algorithm, it can be concluded that, from 30 measured values, there were eliminated 4 values $(13,33 \%$ values - presented in Fig. 7$)$; the repartition of the eliminated and kept values for each of the polyamides tested is presented in Fig. 8. So, in the case of 27 sets of values there where used for computing the average mean all 5 values from the set, in two cases there were used 4 values from the set and in this case the mean was computed by using only 3 values from the set of 5 measured values set is presented in Fig. 9.

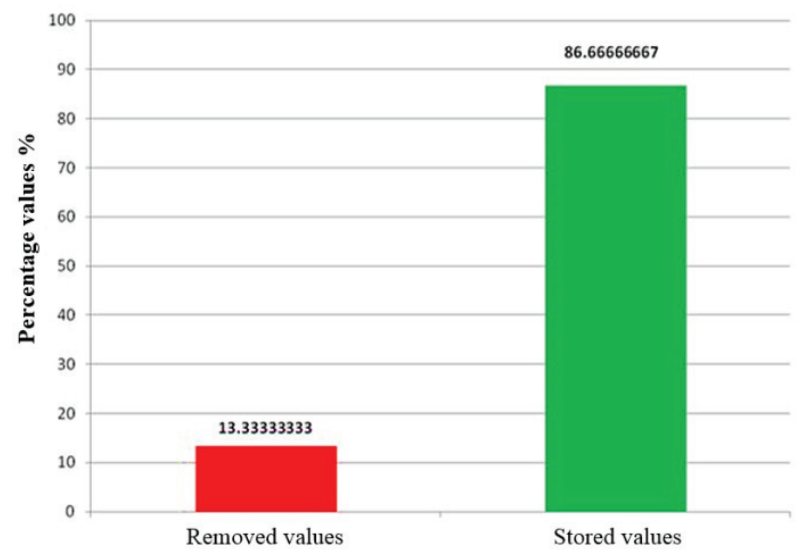

Fig. 7. The overall number used in data processing for PA66, PA46, Teflon PA46.

The friction coefficient values before and after

\footnotetext{
*Corresponding author: radu.papuc@unitbv.ro
} 
applying the modified Thompson $\tau$ technique, in the cases where measured values were eliminated are presented in Fig. 10.

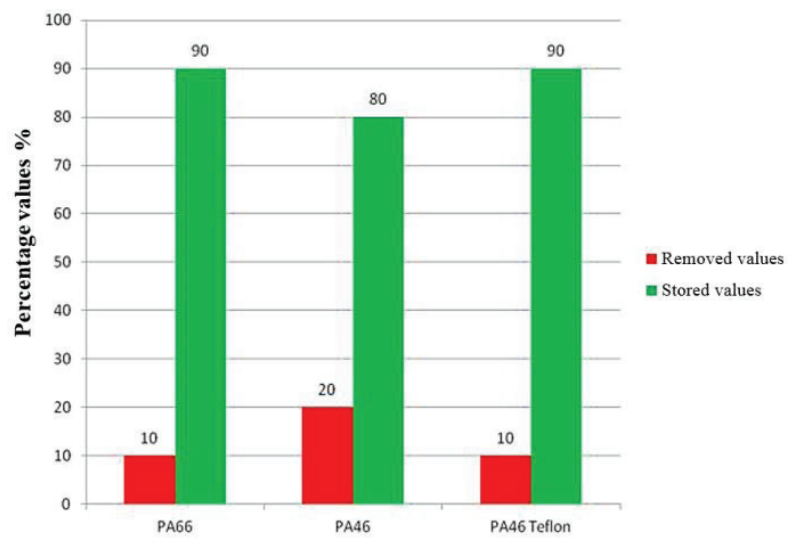

Fig. 8. Repartition of the overall number of values used on tested polyamides.

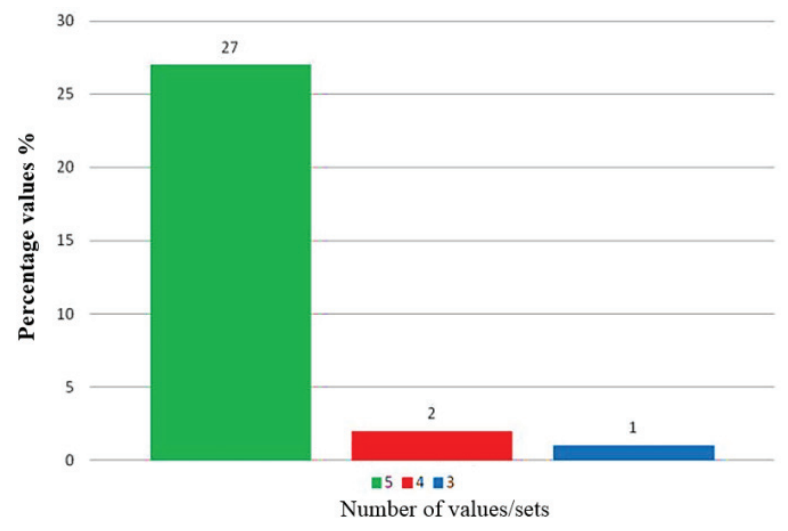

Fig. 9. The number of values/set used for calculating the average mean.

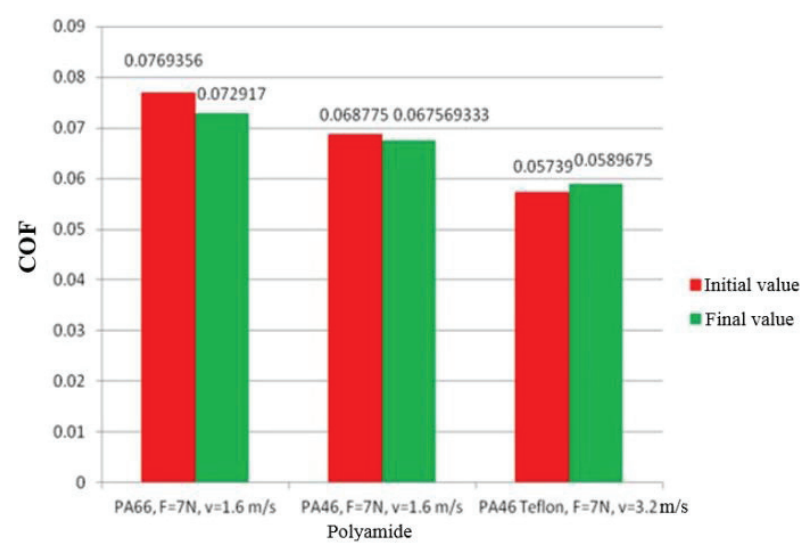

Fig. 10. The friction coefficient before and after applying the modified Thompson $\tau$ technique.

\section{Conclusions}

The differences between the friction coefficient values before and after applying the modified Thompson $\tau$ technique are not big; theses differences can be seen in percentage, for every material.

As it can be seen in Fig. 11, the percentage differences in the case of friction coefficients determined before and after applying the modified Thompson $\tau$ technique, are quite small (as far as 5\%), but they give compliance for the final data, in accordance with the specialty literature from the researched field - document Measurement Uncertainty (American Society of Mechanical Engineers - ASME, 1998).

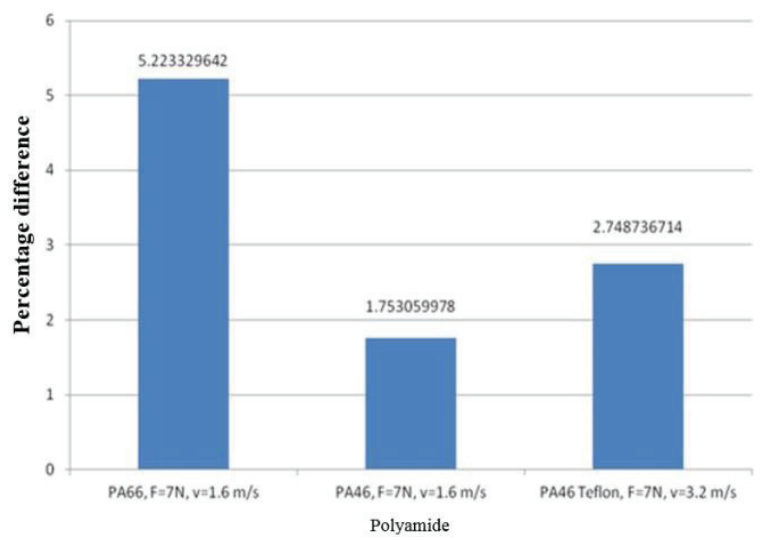

Fig. 11. The percentage difference between the friction coefficients before and after applying the modified Thompson $\tau$ technique.

\section{References}

[1] T. D. Mehnmet, D. Hayrettin, Y. Rifat, "Investigation of temperature effects on tribological properties of glass fiber and MoS2 reinforced PA6.6 and PA66 journal bearings," In: Journal of materials science and engineering, No. A 3 (11), 2013, pp. 738 -742 .

[2] M. W. Shin, S. S. Kim, H. Jang, "Friction and wear of polyamide 66 with different weight average molar mass," In: Tribology letters, No. 44 (2), p. 151 - 158, 2011.

[3] J. Van Ruiten, R. Proost, M. Meuwissen, "How the choise of the polyamide type in timing chains tensioning systems affects the $\mathrm{CO} 2$ emission and fuel economy of internal combustion engines," In Presentation at VDI Veetiltrieb un Zylinderkopf 2012, November 28th, 2012.

[4] K. Randall, B. R. Antoni, "Rolling bearing element diagnostics A tutorial," Journal of Mechanical Systems and Sygnal Processing, Elsevier Publishing House, nr.25, 2011, p. 485-520.

[5] R. Velicu, M. T. Lateș, G. Moldovean, "Loading Cases and Forces on Azimuthal Solar Tracking Systems with Linear Actuators," Proceedings of SYROM 2009, Braşov, Romania, p. 723-733.

[6] A. J. Wheeler, A. R. Ganji, "Introduction to Engineering Experimentation," Third Edition. Pearson Education Publishing House, 2010 